\title{
Penile Prosthesis Infection: Myths and Realities
}

\author{
Kevin J. Hebert ${ }^{(i)}$, Tobias S. Kohler ${ }^{(i)}$ \\ Department of Urology, Mayo Clinic, Rochester, MN, USA
}

Penile prosthesis infection is the most significant complication following prosthesis implant surgery leading to postoperative morbidity, increased health care costs, and psychological stress for the patient. We aimed to identify risk factors associated with increased postoperative penile prosthesis infection. A review of the literature was performed via PubMed using search terms including inflatable penile prosthesis, penile implant, and infection. Articles were given a level of evidence score using the 2011 Oxford Centre for Evidence-Based Medicine Guidelines. Multiple factors were associated with increased risk of post-prosthesis placement infection (Level of Evidence Rating) including smoking tobacco (Level 1), CD4 T-cell count $<300$ (Level 4), Staphylococcus aureus nasal carriage (Level 2), revision surgery (Level 2), prior spinal cord injury (Level 3), and hemoglobin A1c level >8.5 (Level 2). Factors with no effect on infection rate include: preoperative cleansing with antiseptic (Level 4), history of prior radiation (Level 3), history of urinary diversion (Level 4), obesity (Level 3), concomitant circumcision (Level 3), immunosuppression (Level 4), age $>75$ (Level 4), type of hand cleansing (Level 1), post-surgical drain placement (Level 3), and surgical approach (Level 4). Factors associated with decreased rates of infection included: surgeon experience (Level 2), "No Touch" technique (Level 3), preoperative parenteral antibiotics (Level 2), antibiotic coated devices (Level 2), and operative field hair removal with clippers (Level 1). Optimization of pre-surgical and intraoperative risk factors is imperative to reduce the rate of postoperative penile prosthesis infection. Additional research is needed to elucidate risk factors and maximize benefit.

Keywords: Erectile dysfunction; Infection; Penile implant; Penile prosthesis; Sexual dysfunction, physiological

This is an Open Access article distributed under the terms of the Creative Commons Attribution Non-Commercial License (http://creativecommons.org/licenses/by-nc/4.0) which permits unrestricted non-commercial use, distribution, and reproduction in any medium, provided the original work is properly cited.

\section{INTRODUCTION}

Inflatable penile prosthesis (IPP) surgery has the highest patient satisfaction among management options for erectile dysfunction (ED) [1-3]. While previous guidelines recommended IPP surgery for men with medically refractory ED, the most recent American Urological Association (AUA) Guideline statement suggests consideration of IPP surgery as a management option regardless of previous attempts at medical management [4]. Outside of postoperative glans ischemia, prosthesis infection is the most feared complication of IPP surgery, which historically, led to substantial perioperative morbidity. Additionally, prosthesis infection places a significant economic burden on our healthcare system with the documented cost of management being six times the cost of original device placement [5]. Significant effort has been taken to identify risk factors, reduce risk, and optimize management of this complication. We aim to comprehensively review existing literature to provide clarity of risk factors and discuss management strategies of prosthesis infection.

Received: Dec 13, 2018 Revised: Jan 28, 2019 Accepted: Feb 5, 2019 Published online Mar 20, 2019

Correspondence to: Tobias S. Kohler iD https://orcid.org/0000-0002-4947-566X

Department of Urology, Mayo Clinic, 200 1st Street SW, Rochester, MN 55905, USA.

Tel: +1-507-284-3983, Fax: +1-507-284-4951, E-mail: Kohler.Tobias@mayo.edu 


\section{MATERIAL AND METHODS}

A review of the literature was performed via PubMed using search terms including: inflatable penile prosthesis, penile implantation, and infection. Articles were given a level of evidence score using the 2011 Oxford Centre for Evidence-Based Medicine guidelines 2011 [6] (Table 1).

\section{PATIENT FACTORS}

\section{Preoperative cleansing with antiseptic}

Utilization of a preoperative antiseptic wash the night prior to surgery has been advocated by some surgeons in an attempt to reduce bacterial volume on the skin. However, large trials $(n>10,000)$ comparing chlorhexidine with a placebo scrub revealed no statistically significant difference in rates of surgical site infections (SSI) $[7,8]$. Likewise, no differences in infection rates were noted between preoperative scrubs using chlorhexidine or bar soap [8]. This represents Oxford level of evidence 1 for no benefit of preoperative antiseptic wash with chlorhexidine. The International Consultation on Sexual Medicine (ICSM) 2016 Guideline makes no definitive recommendations regarding preoperative site cleansing protocol due to a level of evidence 4 [9].

\section{Smoking status}

The negative effects of smoking tobacco in the immediate postoperative setting have been well documented [10]. A meta-analysis of 140 cohort studies by Sørensen [11] revealed a adjusted odds ratio (OR) of 1.79 for postoperative SSI in smokers versus non-smokers. Likewise, an elevated OR of 3.60 (necrosis), 2.07 (wound dehiscence), and 2.27 (wound complications) was noted in smokers versus non-smokers. A reduction in SSI $(\mathrm{OR}=0.43)$ was identified if patients quit smoking at least 4 weeks preoperatively. As a meta-analysis with a large patient volume $(n=479,150)$, this study represents level of evidence 1, for increased risk of SSI with perioperative smoking tobacco use. Cessation of smoking

Table 1. Perioperative risk factors of IPP infection

\begin{tabular}{|c|c|c|}
\hline Preoperative/intraoperative factor & Implication on infection risk & Level of evidence \\
\hline Preoperative cleansing with antiseptic & No effect on infection risk & 1 \\
\hline Smoking status & Increased risk with active smoking & 1 \\
\hline HIV status & Increased risk if CD4 count $<300$ & 4 \\
\hline Staphylococcus aureus nasal carriage & Increased risk of infection & 2 \\
\hline Preoperative treatment of S. aureus & Decreased risk of infection & 2 \\
\hline Revision surgery & Increased risk of infection & 2 \\
\hline HbA1c level & Increased risk if $\mathrm{HbA} 1 \mathrm{c}>8.5$ & 2 \\
\hline History of pelvic radiation & No effect on infection risk & 3 \\
\hline History of urinary diversion & No effect on infection risk & 4 \\
\hline Spinal cord injury & Increased infection risk & 3 \\
\hline Obesity & No effect on infection risk & 3 \\
\hline Concomitant circumcision & No effect on infection risk & 3 \\
\hline Immunosuppression & No effect on infection risk & 4 \\
\hline Age $>75$ years & No effect on infection risk & 4 \\
\hline Preoperative urine culture & Decreased risk of infection if negative & 5 \\
\hline Method of preoperative hair removal & Decreased risk of infection using clippers & 1 \\
\hline Operative site scrub & Decreased risk of infection with chlorhexidine & 1 \\
\hline Surgeon hand scrub type & No effect on infection risk & 1 \\
\hline Antibiotic impregnated/coated implant & Decreased risk of infection & 2 \\
\hline Perioperative parenteral antibiotics & Decreased risk of infection & 2 \\
\hline Postoperative drain placement & No effect on infection risk & 3 \\
\hline "No Touch" technique & Decreased infection risk & 3 \\
\hline Surgeon experience & Decreased infection risk with more experience & 2 \\
\hline Surgical approach & No effect on infection risk & 4 \\
\hline
\end{tabular}

IPP: inflatable penile prosthesis, HIV: human immunodeficiency virus, HbA1c: hemoglobin A1c. 
prior to surgery is ideal, however, patients should have quit at least 4 weeks prior to their operative case as increased lung secretions in the immediate post-cessation period create an increased risk of lung related infections [11].

\section{Human immunodeficiency virus status}

Human immunodeficiency virus (HIV) is a disease that devastates the adaptive immune system of the host by slowly decreasing CD4 T-cell counts and subsequently increasing the risk of opportunistic infections. Urologic specific studies evaluating the effect of HIV status on SSI risk are lacking. A meta-analysis of 402 HIV-positive patients undergoing orthopedic surgery failed to show a statistically significant increase in postoperative SSI, however, most of the comprising studies were low volume $(\mathrm{n}<100)$ and had missing follow-up data [12]. Anecdotally, one would hypothesize that surgical infection risk would be more closely related to CD4 T-cell counts rather than a patient's isolated HIV status as is the case with nosocomial infection risk. In a small case series of patients $(n=64)$ undergoing orthopedic surgery, an increased rate of postoperative infection was noted in patients with CD4 T-cell counts $<300$ [13]. Thus, we believe there is level 4 evidence that CD4 T-cell counts $<300$ increases infection risk, but that diagnosis of HIV in itself does not.

\section{Staphylococcus aureus nasal carriage}

Staphylococcus aureus is a natural component of human skin flora. However, increasing antibiotic resistance is a major concern since patients undergoing IPP placement have methicillin-resistant Staphylococcus aureus (MRSA) inoculation rates higher than the national average [14]. A large prospective study of patients undergoing orthopedic surgery identified nasal carriage of $S$. aureus at time of surgery as a positive risk factor for the development of S. aureus SSI [15]. However, in the majority of $S$. aureus nasal carriers, the strain identified in the SSI was not concordant with the strain localized to the nares preoperatively [15]. The cause for this conflicting finding is unclear; however, one hypothesis is that patients are likely inoculated by more than one strain of MRSA. A level of evidence of 2 for increased postoperative infection risk is designated due to its large sample size and prospective cohort style.

\section{Preoperative treatment of nasal \\ Staphylococcus aureus}

Although S. aureus has been associated with a higher rate of infection, no study has evaluated for this risk in urologic surgery. A multi-institutional, prospective study found decreased rates of SSI in patients treated with chlorhexidine scrub and mupirocin for 5 days versus placebo, $3.4 \%$ and $7.7 \%$, respectively. No effect in all-cause mortality was identified [16]. A level of evidence rating 2 is recommended based on the large, randomized, prospective nature of this trial. In our practice, we employ this pre-treatment in patients who have a history of previous staphylococcal infection.

\section{Revision surgery}

Surgical failure can be attributed to device malfunction and/or infection. IPP revision surgery, whether due to either cause, comes with increased risk of postoperative infection. Estimates of infection rates following revision surgery have been as high as $10.0 \%$ to $13.3 \%$ compared to $0.46 \%$ to $2.00 \%$ in virgin cases [17-21]. Interestingly, during IPP revision surgery for device failure, Henry et al [22] found positive bacterial cultures on $70 \%$ of clinically uninfected devices suggesting biofilms are a significant source of infection risk with revision surgery. In a small, retrospective series $(n=44)$, Montgomery et al [23] noted a step-wise increase in rate of postoperative infection after stratifying patients by the number of prior IPP surgeries: 1 (6.8\%; 3/44), 2 (18.2\%; 4/22), 3 (33.3\%; 4/12), 4 (50.0\%; 4/8), and 5 (100\%; $2 / 2)$. The increased incidence of postoperative infection following revision surgery is likely multifactorial with scar formation, reduced host resistance, and biofilms each playing a role. Assessment of biofilm formation and organism speciation at time of device explant for clinical infection has shown predominance of gram positive organisms, most commonly coagulase negative Staphylococcus [18,21,24,25]. However, more recent multi-institutional data by Gross et al [26] evaluating culture results at time of clinically infected device explantation or Mulcahy salvage showed no growth in $33 \%$ of cases, gram positive isolates in $73 \%$ of cases, and gram negative isolates in $39 \%$ of cases. Candida (11.1\%), anaerobes (10.5\%), and MRSA (9.2\%) accounted for one third of positive cultures. Furthermore, preoperative antibiotics provided adequate coverage in only $62 \%$ to $86 \%$ of cases based on culture results at time of explant, suggesting that broadened antibiotic prophylaxis 
and review of AUA/European Association of Urology guidelines is necessary [26]. There is level 2 evidence of increased risk with revision surgery based on the number of separate studies with concordant findings, their large sample size, and prospective nature.

\section{Diabetes/hemoglobin Alc level}

Diabetes is a known cause of multi-organ dysfunction often effecting small, end-arteries in the eyes, extremities, and penis. While, diabetes is recognised to impact postoperative wound healing and infection risk, the pathophysiology of this process is complex and likely multifactorial. Decreased fibroblast proliferation and impaired polymorphonuclear neutrophil cell function have been found to play a significant role $[27,28]$. The effect of diabetes on postoperative prosthesis infection risk has produced differing results in the literature. Mulcahy and Carson [29] found a significantly increased rate of postoperative infections in diabetics versus non-diabetics, $1.88 \%$ and $1.53 \%$, respectively. Yet, other retrospective studies did not confirm an increased risk of infection [17,30]. Varied results could exist due to the extensive range of glucose control.

Hemoglobin A1c (HbA1c) is a marker for glycemic control over a 3 month period with elevation in $\mathrm{HbA1c}$ being associated with poor control. The effect of perioperative HbA1c levels on postoperative IPP infection rates have varied. In a small prospective study ( $\mathrm{n}=90)$, Bishop et al [31] found poorly controlled diabetics (HbA1c $>11.5$ ) more likely to have a postoperative infection than properly controlled diabetics (31\% versus $5 \%$, respectively). A larger prospective study of 389 IPPs by Wilson et al [32], failed to identify a statistically significant correlation between $\mathrm{HbA1c}$ and infection. In a recent multi-center, prospective study of 902 implants (76\% malleable), patients were stratified into $\mathrm{HbA1C}$ groups of $<6.5 \%, 6.5 \%$ to $7.5 \%, 7.6 \%$ to $8.5 \%, 8.6 \%$ to $9.5 \%$, and $>9.5 \%$, with postoperative infection rates of $1.5 \%$, $6.5 \%, 14.7 \%$, and $22.4 \%$, respectively. Using a receiver operating characteristic curve, a threshold HbA1c level of $8.5 \%$ was identified to indicate increased postoperative risk of IPP infection [33]. We would confer Oxford level of evidence rating 2 for increased risk of postoperative penile prosthesis infection in patients with a perioperative $\mathrm{HbA1c}>8.5$. Future research should focus on perioperative glucose control and its effect on infection risk.

\section{History of radiation therapy}

Pelvic radiation, commonly utilized in the treatment of prostate cancer, has been shown to increase risk for ED in a delayed fashion [34]. Prior radiation been has been associated with an increased risk of adverse outcomes in artificial genitourinary sphincter population [35-37]. However, radiated sphincter infection data is confounded by higher rates of urethral cuff erosion which is linked with infection. In the IPP literature, no contemporary studies have shown a statistically significant difference in infections rates [18,38,39]. Thus, there is level 3 evidence that infection rates are not affected by radiation.

\section{History of urinary diversion}

In a recent retrospective review of 80 patients undergoing IPP placement following radical cystectomy with urinary diversion, 4 patients $(5.0 \%)$ developed postoperative prosthesis infection. While this represents a slightly higher infection rate than contemporary studies with virgin cases, Loh-Doyle et al [40] display the safety and feasibility of penile prosthesis implantation in this difficult patient population. Due to a lack of randomized controlled trials (RCTs), urinary diversion following radical cystectomy not effecting postoperative infections rates is currently level of evidence 4 .

\section{Spinal cord injury}

Spinal cord injuries (SCI) are a common etiology of sexual dysfunction which can lead to significant psychological morbidity [41]. Bowel and bladder dysfunction are commonly seen in the SCI population which can increase the risk of both asymptomatic bacteruria and urinary tract infections. SCI also often require intermittent catheterization. We have experienced a few 'infections' which ultimately were spawned from aggressive catheterization and urethral injury at the distal urethra. In addition, SCI patients are often insensate in their genital region, leading to higher device erosion rates (especially with malleable devices). Evaluation of prosthesis infection risk in the SCI population has not surprisingly produced varied results likely due to these confounders. Two large studies found a statistically significant increase in postoperative infection rates in patients with SCI [18,42]. However, in two smaller studies, an increased postoperative infection rate was not found $[17,43]$. Due to the higher power of the larger volume studies [18,42], we recommend level 3 
evidence for increased risk of postoperative infection in the SCI population.

\section{Obesity}

Both the prevalence and degree of obesity are rising in the United States [44]. Obesity has significant implications in the pathophysiology of metabolic syndrome and other systemic diseases. Likewise, it has been associated with an increased risk of SSI in two separate meta-analyses in colorectal and orthopaedic surgery $[45,46]$. However, its cross-over applicability to penile prosthesis surgery is uncertain as no difference in postoperative infection rates were seen by Wilson and Delk [18] when controlled for diabetes. Wound healing problems and seroma formation are common in obese patients for incisions in areas of excess fat. However, penoscrotal incisions are spared these issues due to the lack of fat in the scrotum. Thus, there is level 3 evidence that obesity does not confer increased risk in penile implants.

\section{Concomitant circumcision}

The consent of pre-pubertal circumcision has been debated. However, the benefits of circumcision include reduced infection rate, decreased risk of penile cancer, and decreased risk of STI transmission [47,48]. Occasionally men will request concomitant circumcision at the time of penile prosthesis implantation which has raised concern for possible increased risk of infection. Three urologic studies assessed the risk of infection with this added procedure. In a series of 34 patients undergoing simultaneous circumcision and prosthesis placement by Thomalla et al [20], none were complicated by infection. This finding was reinforced by Wilson and Delk [18] which revealed no increase in infections with a circumcision incision. Additional evidence in a recent study by Weinberg et al [49] describing 200 men who underwent circumcision incision approach for IPP placement, reported postoperative infection in 3 patients. $53 \%$ of the patients also had simultaneous circumcision; however, it is unclear if any association was present in the 3 postoperative infections. The most recent study by Weinberg et al [49] provides additional contemporary evidence that incision type and concurrent circumcision do not increase the risk of postoperative infection, thus, we have applied level of evidence 3.

\section{Immunosuppression}

The immunosuppressed patient poses significant challenges in the perioperative period due to concern for poor wound healing and susceptibility to infection. Review of IPP literature suggests varied results with Sidi et al [50] reporting no infections in 13 immunosuppressed patients undergoing penile prosthesis placement. However, Wilson and Delk [18] encountered a postoperative infection in 5 of 10 patients on chronic steroid therapy, but no infectious complications in 3 renal transplant patients. A more recent study by Cuellar and Sklar [51] found similar rates of infection among 46 previous organ transplant patients undergoing IPP placement compared to non-transplant patients. This data would suggest Oxford level of evidence 4 for no increased risk of postoperative infection among the immunosuppressed population.

\section{Age $>75$ years}

In 2010, 17 million men in the United States were 65 years of age or older which is projected to increase to 39.9 million by 2050 [52]. Likewise, an increase in men 85 years of age or older is projected to total 4.2 million [52]. We would expect an increase demand for penile prosthesis in the $>75$ year old population. Immunological response to infectious agents decreases with age due to changes in T-cell response [53], raising the question if increased age increases risk for postoperative IPP infection. Chung et al [54] did not report any postoperative infections in 30 men aged over 75 years. Additionally, Wilson and Delk [18] did not find age to be a risk factor in his $>1,000$ patient experience reported in 1995, although an age range was not reported. Due to the non-controlled cohort style of the literature available, we would confer a level of evidence 4 for no increased risk of infection with age, although we expect as our population continues to age, better data will become available.

\section{SURGICAL FACTORS}

\section{Preoperative urine culture}

The product label for both Coloplast (Minneapolis, MN, USA) and Boston Scientific (Minnetonka, MN, USA) state IPP placement is contraindicated in patients with active urogenital infections. In addition, the AUA anti-microbial prophylaxis best practice guidelines state that attempts should be made to sterilize 
the surgical field prior to procedures [55]. Thus, many surgeons routinely obtain preoperative urine cultures prior to IPP placement and actively attempt to sterilize the urine in men who perform clean intermittent catheterization. However, in a large, single-surgeon, retrospective review of urologic prosthetic placement (artificial genitourinary sphincter placement [ $\mathrm{n}=337]$ and IPP [ $\mathrm{n}=367]), 41 \%$ of patients did not have a preoperative urine culture. No statistically significant difference in rate of postoperative infection rates were identified between those who did and did not have preoperative urine cultures [56]. This study was underpowered to detect a true difference and did not focus on high risk patients. Overall, level 5 evidence (expert opinion) advocates pre-procedure urine culture, especially for men who are symptomatic or high risk.

\section{Hair removal}

Removal of body hair preoperatively is usually preformed with clippers or razor. Due to skin ruggae, scrotal skin poses a difficult surface for hair removal. Multiple studies have addressed methods of hair removal and SSI risk. In a Cochrane review of six trials addressing hair removal before surgery versus no hair removal, no statistically significant difference was found in rate of postoperative infection. However, use of a razor for hair removal compared to use of clippers was associated with an increase in postoperative infection (relative risk $=2.09,95 \%$ confidence interval=1.15-3.80). No statistically significant difference in infection rates were seen whether hair was removed the day before surgery versus in the operating room [57]. The 2016 ICSM leaves the use of a razor versus clippers to the discretion of the performing surgeon [9]. Based on the large Cochrane review showing decreased risk of infection with use of clippers, this would represent level 1 evidence for preparation of infrapubic incisions. No data has evaluated differing modalities of scrotal skin prep prior to penoscrotal incisions.

\section{Operative site scrub}

The majority of cultures obtained from postoperative prosthesis infections grow gram positive organisms including coagulase negative Staphylococcus, suggesting likely skin contamination. Thus, achieving a sterile field is of utmost importance. Historically, a povidone-iodine based scrub was used. However, a recent prospective, randomized trial in patients un- dergoing genitourinary prosthesis surgery assessed which antiseptic scrub reduced bacterial skin flora the best. One hundred patients were randomized into two arms, chlorhexidine and povidone-iodine. Pre-scrub and post-scrub culture swabs were performed. Use of a providone-iodine scrub had an increased rate of postscrub positive skin culture compared to chlorhexidine, $32 \%$ versus $8 \%(\mathrm{p}=0.0091)$, respectively [58]. Additionally, 2 large randomized trials both showed a statistically significant reduced postoperative infection rate with the use of chlorhexidine preoperative scrub versus povidone-iodine scrub [59,60]. Likewise, the 2016 ICSM guidelines recommend use of an alcohol based skin prep prior to IPP placement, level of recommendation A [9]. Due to concordant findings in multiple, randomized prospective trials, we confer level of evidence of 1 for use of chlorhexidine for pre-surgical scrub.

\section{Surgeon hand cleansing}

Double-gloving during IPP surgery is an integral part of our practice due to the risk of glove perforation and subsequent device contamination from skin flora. Significant emphasis has been directed towards determining the optimal pre-surgical hand scrub. A recent Cochrane Review of 14 trials on the topic in 2016 found no evidence of superiority of any particular scrub method [61]. The Consultation on Sexual Medicine 2016 guidelines does not make a recommendation on surgeon hand scrub [9]. Based on a Cochrane Review of 14 prospective trials, we suggest no difference in postoperative infection rates based on type of surgical hand scrub utilized, level of evidence 1.

\section{Antibiotic impregnated/coated implant}

Design improvements in prosthetic devices, most importantly antibiotic impregnation/coating, have led to a significant reduction in postoperative prosthesis infection. Boston Scientific utilizes InhibiZone ${ }^{\circledR}$, a device impregnation technology with minocycline/rifampin, while Coloplast utilizes a hydrophilic coating which absorbs any aqueous antibiotic solution left to surgeon discretion. In a systematic review of 14 trials $(>9,000$ patients) of coated versus non-coated prosthesis, Mandava et al [62], found a higher rate of postoperative infection in non-coated devices versus coated devices (2.32 versus $0.89 \%$; $\mathrm{p}<0.01$ ). Likewise, Mandava et al [62] evaluated which antibiotic combination was most efficacious against postoperative infection. Vancomycin/ 
gentamicin, minocycline/rifampin, and rifampin/gentamicin combinations were compared with postoperative infection rates of $4.42 \%, 0.63 \%$, and $0.55 \%$, respectively. As previously discussed, biofilms play a significant role in the pathophysiology of prosthesis infection. A recent study by Jani et al [63] evaluated the impact of antibiotic coated devices on bacterial isolates at time of revision surgery. This is a combined retrospective, now prospective, non-randomized multi-center study assessing the difference in bacterial culture results of prosthesis devices at time of explantation. There was an increased rate of bacterial growth on non-coated prostheses versus coated prostheses in devices removed for mechanical failure (63.9\% versus $42.7 \%$, $\mathrm{p}=0.004)$. Notably, a decrease in the percentage of coagulase negative Staphylococcus was seen in devices coated with antibiotics. A level of evidence of 2 is given for an increased risk of device infection when not coated with antibiotics due to the results of a large, retrospective meta-analysis.

\section{Preoperative parenteral antibiotics}

Currently no RCTs exist in urologic prosthetic literature comparing the benefit of perioperative antibiotics. However, a clear benefit is identified with extrapolation of data from RCTs evaluating the efficacy of perioperative antibiotics with orthopaedics implant surgery and mesh placement in general surgery $[64,65]$. The ICSM for Penile Prosthesis Surgery provides a grade B recommendation for perioperative antibiotic administration with gram positive and gram negative coverage prior to surgical incision in IPP surgery, however, no recommendations on specific antibiotics are provided [9]. The AUA perioperative anti-microbial guidelines recommend first line prophylaxis with an aminoglycoside plus 1st/2nd generation cephalosporin or vancomycin. Alternative regimens include: Ampicillin/Sulbactam, Ticarcillin/Clavulanate, or Pipercillin/Tazobactam [55]. However, adjustments to perioperative antibiotic recommendations may be necessary following a seminal paper by Gross et al [26] showing AUA guideline based perioperative antibiotics covered prosthesis culture isolates only $62 \%$ to $86 \%$ of time. A level of evidence of 2 for the use of perioperative antibiotics is noted due to multiple RCTs studies.

\section{Postoperative drain placement}

The gravity dependent nature of the scrotum can lead to postoperative hematoma formation which can delay device usage, increase postoperative pain, and increase postoperative infection risk. Manuevers to reduce hematoma formation including the Henry Mummy wrap ${ }^{\mathrm{TM}}$ [66], scrotal elevation, jock strap use, and placement of a 10 French Blake drain have been reported. Concern for increased risk of device infection with placement of a Blake drain has been raised. Relatively limited data is available in the literature, however, one large, multi-center study of 425 patients undergoing IPP surgery with postoperative drain utilization found a similar infection rate (3.3\%) compared to when no drain was left in place [67]. Based on the available literature the use of a drain not effecting postoperative infection rates is currently Oxford level of evidence 3 .

\section{8. "No Touch" technique}

Eid et al [21] published his experience with the "No Touch" technique in 2012 which aimed to eliminate prosthesis-skin contact by using a plastic drape to cover the surgical field at time of prosthesis insertion. The concern for infection risk with skin contact is logical with the majority of prosthesis infection cultures showing coagulase negative Staphylococcus [22,26,63]. Eid et al [21] reported 3,342 penile implants cases separated into 3 groups: without infection retardant, with infection retardant, and with infection retardant/no-touch technique with reported infection rates of 5.3\%, 1.99\%, and $0.44 \%$, respectively. Utilization of the "No Touch" technique and its reduction in postoperative infection risk is Oxford level of evidence of 3.

\section{Surgeon experience}

Surgeon experience and its relation to adverse outcomes has been shown to be a significant factor in studies across multiple surgical sub-specialties [68-70]. A recent practice pattern analysis revealed that lowvolume surgeons (providers implanting no more than 4 IPPs yearly) performed $75 \%$ of IPP surgeries in the United States [71]. Historically, academic institutions have implanted significant numbers of penile prostheses. The experience of surgeons-in-training and their participation in IPP implantation did not increase the rate of postoperative IPP infection in a recent retrospective review, of a high-volume implanter in an academic setting [72]. A review of the New York Statewide Planning and Research Cooperative System database 
revealed 14,969 men who underwent IPP placement between 1995 and 2014. The reoperation rate for infection was $2.5 \%$. On multivariable analysis, statistically significant differences in postoperative infections rates were noted depending on surgeon yearly IPP volume. Men undergoing IPP surgery by surgeons in the lowest quartile ( $0-2$ cases per year), lower-mid quartile (3-7 cases per year), and upper-mid quartile (8-31 cases per year) were $2.5,2.4$, and 2.1 times more likely to have a postoperative infection compared to patients undergoing IPP placement by surgeons in the highest quartile of IPP surgery volume ( $>31$ cases per year) [73]. Surgeon experience has a significant impact on prosthesis infection risk, level of evidence of 2 .

\section{Surgical approach}

Utilization of a penoscrotal or infrapubic incision are the two most common approaches to penile prosthesis surgery. A less common approach involves a circumcision incision with degloving of the penis. The type of surgical approach and its effect on infection rates has been explored with Garber and Marcus [30] comparing infection rates between the penoscrotal and infrapubic approaches. In his study of 360 primary implants, 4 of 139 implants (2.9\%) performed via infrapubic approach became infected compared to 2 of 221 (0.9\%) via penoscrotal approach. However, this did not achieve statistical significance $(p=0.18)$. Weinberg et al [49] reported a sub-coronal approach with no-touch technique in 200 men with postoperative infection noted in 3 patients (1.5\%). The 2016 ICSM makes no recommendation of surgical approach based on infection risk [9]. Due to the retrospective, case-series nature of the involved studies, this represents level of evidence of 4 .

\section{Salvage techniques}

Historically, virgin implant cases carry a risk of infection of $0.5 \%$ to $3 \%$ while revision cases can have infections rates ranging from $10 \%$ to $13 \%$ [17-21]. Until the mid-1990s, standard of care for clinically infected penile prostheses included antibiotic therapy and immediate device removal with delay in re-implantation after approximately 6 months. This resulted in corporal fibrosis, penile shortening, increased difficulty of IPP reinsertion, and reduced patient satisfaction [74]. In 1996, Brant/Mulcahy described a new protocol for immediate replacement of infected IPPs which involved complete device removal, serial wound washout, followed by re-implantation of a new IPP [75]. Patients with device erosion and frank purulence were excluded. Post salvage, only 1 of 11 patients developed postoperative infection at mean follow-up of 21 months [75]. Similar results were shown in 2000 with Mulcahy [76] reporting no evidence of infection in 45 of 55 patients (82\%) at mean follow-up 35 months. Additional studies have reported similar success with the Mulcahy salvage technique [77-79]. In 2016, Gross et al [25] reported a decrease in postoperative infection rates in a retrospective, multi-institutional experience with 54 patients undergoing Mulcahy salvage with immediate replacement with a malleable device. Of the 54 patients, $93 \%$ were infection free at follow-up ranging from 1 to 84 months. Notably, only $31 \%$ of patients elected to convert from a malleable device to an inflatable device at a later date. Despite the success of the Mulcahy salvage procedure, conservative management of a localized infection may be a viable option based on a recent study by Habous et al [80]. Thirty-seven patients (all diabetics) with localized post-implant infections were treated with antibiotic therapy alone. Exclusion criteria included temperature $>37.5^{\circ} \mathrm{C}$, white blood cell $>13,000$, or signs of sepsis. Four of 37 patients required device removal due to failed antibiotic therapy at a mean time-point of 75 days. Two additional patients required device removal due to persistent pain. However, 31 of 37 patients (83.8\%) had complete clinical resolution of infection with a mean time of antibiotic therapy of 49 days [80].

In review, immediate explantation of penile prostheses following local infection with immediate device replacement should be considered standard of care when performed at a high-volume center. However, in carefully selected patients, prolonged conservative management with antibiotic therapy may play an increased role especially when considering differences in cost. Due to the retrospective, non-randomized nature of these studies, the current salvage therapies presented represent level of evidence of 3 .

\section{CONCLUSIONS}

Postoperative prosthesis infection leads to significant morbidity which places strain on our healthcare system [5]. Much effort has been directed towards identifying risk factors and methods to reduce the risk of prosthesis infection. Modification of preoperative/ 
perioperative risk factors and adjustments in prosthesis design have reduced infection rates significantly in the last decade. Although excellent progress has been made, continued research on the effects of obesity, diabetes, antimicrobial coverage, and device design on postoperative infection will be paramount as increased prosthesis demand is encountered due to the rising age of our patient population.

\section{Disclosure}

Dr. Tobias S. Kohler acts as a consultant to both Coloplast and Boston Scientific. Kevin J. Hebert has no disclosures.

\section{Author Contribution}

Conception or design of the work: Hebert KJ, Kohler TS. Data collection: Hebert KJ. Data analysis and interpretation: Hebert KJ, Kohler TS. Drafting the article: Hebert KJ. Critical revision of the article: Hebert KJ, Kohler TS. Final approval of the version to be published: Kohler TS.

\section{REFERENCES}

1. Bettocchi C, Palumbo F, Spilotros M, Lucarelli G, Palazzo S, Battaglia M, et al. Patient and partner satisfaction after AMS inflatable penile prosthesis implant. J Sex Med 2010;7:304-9.

2. Carson CC, Mulcahy JJ, Govier FE. Efficacy, safety and patient satisfaction outcomes of the AMS 700CX inflatable penile prosthesis: results of a long-term multicenter study. AMS 700CX Study Group. J Urol 2000;164:376-80.

3. Lindeborg L, Fode M, Fahrenkrug L, Sønksen J. Satisfaction and complications with the $\operatorname{Titan}^{\circledR}$ one-touch release penile implant. Scand J Urol 2014;48:105-9.

4. Burnett AL, Nehra A, Breau RH, Culkin DJ, Faraday MM, Hakim LS, et al. Erectile dysfunction: AUA guideline. J Urol 2018;200:633-41.

5. Montague DK, Angermeier KW, Lakin MM. Penile prosthesis infections. Int J Impot Res 2001;13:326-8.

6. OCEBM Levels of Evidence Working Group. The Oxford levels of evidence 2 [Internet]. Oxford: Oxford Centre for Evidence-Based Medicine; 2011 [cited 2018 Oct 25]. Available from: https://www.cebm.net/index.aspx?o=5653.

7. Chlebicki MP, Safdar N, O'Horo JC, Maki DG. Preoperative chlorhexidine shower or bath for prevention of surgical site infection: a meta-analysis. Am J Infect Control 2013;41:16773.

8. Webster J, Osborne S. Preoperative bathing or showering with skin antiseptics to prevent surgical site infection. Cochrane Database Syst Rev 2015;(2):CD004985.

9. Levine LA, Becher EF, Bella AJ, Brant WO, Kohler TS, Martinez-Salamanca JI, et al. Penile prosthesis surgery: current recommendations from the International Consultation on Sexual Medicine. J Sex Med 2016;13:489-518.

10. Turan A, Mascha EJ, Roberman D, Turner PL, You J, Kurz A, et al. Smoking and perioperative outcomes. Anesthesiology 2011;114:837-46.

11. Sørensen LT. Wound healing and infection in surgery. The clinical impact of smoking and smoking cessation: a systematic review and meta-analysis. Arch Surg 2012;147:373-83.

12. Kigera JW, Straetemans M, Vuhaka SK, Nagel IM, Naddumba $\mathrm{EK}$, Boer K. Is there an increased risk of post-operative surgical site infection after orthopaedic surgery in HIV patients? A systematic review and meta-analysis. PLoS One 2012; 7:e42254.

13. Guild GN, Moore TJ, Barnes W, Hermann C. CD4 count is associated with postoperative infection in patients with orthopaedic trauma who are HIV positive. Clin Orthop Relat Res 2012;470:1507-12.

14. Silverstein A, Henry GD, Delk JR, Wilson SK, Donatucci CF. Nasal carriage of Staphylococcus aureus as a potential risk factor for infection after penile prosthesis placement. Int J Impot Res 2002;14:S61.

15. Berthelot P, Grattard F, Cazorla C, Passot JP, Fayard JP, Meley $\mathrm{R}$, et al. Is nasal carriage of Staphylococcus aureus the main acquisition pathway for surgical-site infection in orthopaedic surgery? Eur J Clin Microbiol Infect Dis 2010;29:373-82.

16. Bode LG, Kluytmans JA, Wertheim HF, Bogaers D, Vandenbroucke-Grauls CM, Roosendaal R, et al. Preventing surgicalsite infections in nasal carriers of Staphylococcus aureus. N Engl J Med 2010;362:9-17.

17. Jarow JP. Risk factors for penile prosthetic infection. J Urol 1996;156:402-4.

18. Wilson SK, Delk JR 2nd. Inflatable penile implant infection: predisposing factors and treatment suggestions. J Urol 1995; 153:659-61.

19. Wilson SK, Zumbe J, Henry GD, Salem EA, Delk JR, Cleves MA. Infection reduction using antibiotic-coated inflatable penile prosthesis. Urology 2007;70:337-40.

20. Thomalla JV, Thompson ST, Rowland RG, Mulcahy JJ. Infectious complications of penile prosthetic implants. J Urol 1987;138:65-7.

21. Eid JF, Wilson SK, Cleves M, Salem EA. Coated implants and "no touch" surgical technique decreases risk of infection in inflatable penile prosthesis implantation to $0.46 \%$. Urology 2012;79:1310-5. 
22. Henry GD, Wilson SK, Delk JR 2nd, Carson CC, Silverstein A, Cleves MA, et al. Penile prosthesis cultures during revision surgery: a multicenter study. J Urol 2004;172:153-6.

23. Montgomery BD, Lomas DJ, Ziegelmann MJ, Trost LW. Infection risk of undergoing multiple penile prostheses: an analysis of referred patient surgical histories. Int J Impot Res 2018;30:147-52.

24. Henry GD, Wilson SK, Delk JR 2nd, Carson CC, Wiygul J, Tornehl C, et al. Revision washout decreases penile prosthesis infection in revision surgery: a multicenter study. J Urol 2005;173:89-92.

25. Gross MS, Phillips EA, Balen A, Eid JF, Yang C, Simon R, et al. The malleable implant salvage technique: infection outcomes after mulcahy salvage procedure and replacement of infected inflatable penile prosthesis with malleable prosthesis. J Urol 2016;195:694-7.

26. Gross MS, Phillips EA, Carrasquillo RJ, Thornton A, Greenfield JM, Levine LA, et al. Multicenter investigation of the micro-organisms involved in penile prosthesis infection: an analysis of the efficacy of the AUA and EAU guidelines for penile prosthesis prophylaxis. J Sex Med 2017;14:455-63.

27. McManus LM, Bloodworth RC, Prihoda TJ, Blodgett JL, Pinckard RN. Agonist-dependent failure of neutrophil function in diabetes correlates with extent of hyperglycemia. J Leukoc Biol 2001;70:395-404.

28. Barbul A, Efron DT, Kavalukas SL. Wound healing. In: Brunicardi FC, Andersen DK, Billiar TR, Dunn DL, Hunter JG, Matthews JB, et al., editors. Schwartz's principles of surgery. 10th ed. New York: McGraw-Hill Education; 2014.

29. Mulcahy JJ, Carson CC 3rd. Long-term infection rates in diabetic patients implanted with antibiotic-impregnated versus nonimpregnated inflatable penile prostheses: 7-year outcomes. Eur Urol 2011;60:167-72.

30. Garber BB, Marcus SM. Does surgical approach affect the incidence of inflatable penile prosthesis infection? Urology 1998;52:291-3.

31. Bishop JR, Moul JW, Sihelnik SA, Peppas DS, Gormley TS, McLeod DG. Use of glycosylated hemoglobin to identify diabetics at high risk for penile periprosthetic infections. J Urol 1992;147:386-8.

32. Wilson SK, Carson CC, Cleves MA, Delk JR 2nd. Quantifying risk of penile prosthesis infection with elevated glycosylated hemoglobin. J Urol 1998;159:1537-9; discussion 1539-40.

33. Habous M, Tal R, Tealab A, Soliman T, Nassar M, Mekawi $\mathrm{Z}$, et al. Defining a glycated haemoglobin (HbA1c) level that predicts increased risk of penile implant infection. BJU Int 2018;121:293-300.

34. Goldstein I, Feldman MI, Deckers PJ, Babayan RK, Krane
RJ. Radiation-associated impotence. A clinical study of its mechanism. JAMA 1984;251:903-10.

35. McGeady JB, McAninch JW, Truesdale MD, Blaschko SD, Kenfield S, Breyer BN. Artificial urinary sphincter placement in compromised urethras and survival: a comparison of virgin, radiated and reoperative cases. J Urol 2014;192:1756-61.

36. Rivera ME, Linder BJ, Ziegelmann MJ, Viers BR, Rangel LJ, Elliott DS. The impact of prior radiation therapy on artificial urinary sphincter device survival. J Urol 2016;195:1033-7.

37. Brant WO, Erickson BA, Elliott SP, Powell C, Alsikafi N, McClung $\mathrm{C}$, et al. Risk factors for erosion of artificial urinary sphincters: a multicenter prospective study. Urology 2014;84:934-8.

38. Dubocq FM, Bianco FJ Jr, Maralani SJ, Forman JD, Dhabuwala $\mathrm{CB}$. Outcome analysis of penile implant surgery after external beam radiation for prostate cancer. J Urol 1997;158:178790 .

39. Loh-Doyle J, Patil MB, Nakhoda Z, Nassiri N, Yip W, Wayne $\mathrm{K}$, et al. Three-piece inflatable penile prosthesis placement following pelvic radiation: technical considerations and contemporary outcomes. J Sex Med 2018;15:1049-54.

40. Loh-Doyle J, Patil MB, Sawkar H, Wayne K, Boyd SD. 3-piece inflatable penile prosthesis placement following radical cystoprostatectomy and urinary diversion: technique and outcomes. J Sex Med 2018;15:907-13.

41. Biering-Sørensen F, Sønksen J. Sexual function in spinal cord lesioned men. Spinal Cord 2001;39:455-70.

42. Collins KP, Hackler RH. Complications of penile prostheses in the spinal cord injury population. J Urol 1988;140:984-5.

43. Radomski SB, Herschorn S. Risk factors associated with penile prosthesis infection. J Urol 1992;147:383-5.

44. Hales CM, Carroll MD, Fryar CD, Ogden CL. Prevalence of obesity among adults and youth: United States, 2015-2016. NCHS Data Brief 2017;(288):1-8.

45. Yuan K, Chen HL. Obesity and surgical site infections risk in orthopedics: a meta-analysis. Int J Surg 2013;11:383-8.

46. Gurunathan U, Ramsay S, Mitrić G, Way M, Wockner L, Myles P. Association between obesity and wound infection following colorectal surgery: systematic review and metaanalysis. J Gastrointest Surg 2017;21:1700-12.

47. Tobian AA, Gray RH, Quinn TC. Male circumcision for the prevention of acquisition and transmission of sexually transmitted infections: the case for neonatal circumcision. Arch Pediatr Adolesc Med 2010;164:78-84.

48. Douglawi A, Masterson TA. Updates on the epidemiology and risk factors for penile cancer. Transl Androl Urol 2017;6:785-90.

49. Weinberg AC, Pagano MJ, Deibert CM, Valenzuela RJ. Sub- 
coronal inflatable penile prosthesis placement with modified no-touch technique: a step-by-step approach with outcomes. J Sex Med 2016;13:270-6.

50. Sidi AA, Peng W, Sanseau C, Lange PH. Penile prosthesis surgery in the treatment of impotence in the immunosuppressed man. J Urol 1987;137:681-2.

51. Cuellar DC, Sklar GN. Penile prosthesis in the organ transplant recipient. Urology 2001;57:138-41.

52. Vincent GK, Velkoff VA. The next four decades: the older population in the United States: 2010 to 2050. Washington: Department of Commerce, Economics and Statistics Administration, U.S. Census Bureau; 2010;25-1138.

53. Ginaldi L, De Martinis M, D’Ostilio A, Marini L, Loreto MF, Martorelli V, et al. The immune system in the elderly: II. Specific cellular immunity. Immunol Res 1999;20:109-15.

54. Chung E, Solomon M, DeYoung L, Brock GB. Clinical outcomes and patient satisfaction rates among elderly male aged $\geq 75$ years with inflatable penile prosthesis implant for medically refractory erectile dysfunction. World J Urol 2014;32:173-7.

55. Wolf JS Jr, Bennett CJ, Dmochowski RR, Hollenbeck BK, Pearle MS, Schaeffer AJ. Best practice policy statement on urologic surgery antimicrobial prophylaxis. J Urol 2008;179:1379-90.

56. Kavoussi NL, Viers BR, Pagilara TJ, Siegel JA, Hofer MD, Cordon $\mathrm{B}$, et al. Are urine cultures necessary prior to urologic prosthetic surgery? Sex Med Rev 2018;6:157-61.

57. Tanner J, Norrie P, Melen K. Preoperative hair removal to reduce surgical site infection. Cochrane Database Syst Rev 2011;(11):CD004122.

58. Yeung LL, Grewal S, Bullock A, Lai HH, Brandes SB. A comparison of chlorhexidine-alcohol versus povidone-iodine for eliminating skin flora before genitourinary prosthetic surgery: a randomized controlled trial. J Urol 2013;189:136-40.

59. Paocharoen V, Mingmalairak C, Apisarnthanarak A. Comparison of surgical wound infection after preoperative skin preparation with $4 \%$ chlorhexidine [correction of chlohexidine] and povidone iodine: a prospective randomized trial. J Med Assoc Thai 2009;92:898-902.

60. Darouiche RO, Wall MJ Jr, Itani KM, Otterson MF, Webb AL, Carrick MM, et al. Chlorhexidine-alcohol versus povidoneiodine for surgical-site antisepsis. N Engl J Med 2010;362:1826.

61. Tanner J, Dumville JC, Norman G, Fortnam M. Surgical hand antisepsis to reduce surgical site infection. Cochrane Database Syst Rev 2016;(1):CD004288.

62. Mandava SH, Serefoglu EC, Freier MT, Wilson SK, Hellstrom WJ. Infection retardant coated inflatable penile prostheses decrease the incidence of infection: a systematic review and meta-analysis. J Urol 2012;188:1855-60.

63. Jani K, Smith C, Delk JR 2nd, Carson CC, Donatucci CF, Cleves MA, et al. Infection retardant coatings impact on bacterial presence in penile prosthesis surgery: a multicenter study. Urology 2018;119:104-8.

64. Boxma H, Broekhuizen T, Patka P, Oosting H. Randomised controlled trial of single-dose antibiotic prophylaxis in surgical treatment of closed fractures: the Dutch Trauma Trial. Lancet 1996;347:1133-7.

65. Yerdel MA, Akin EB, Dolalan S, Turkcapar AG, Pehlivan M, Gecim IE, et al. Effect of single-dose prophylactic ampicillin and sulbactam on wound infection after tension-free inguinal hernia repair with polypropylene mesh: the randomized, double-blind, prospective trial. Ann Surg 2001;233:26-33.

66. Henry GD. The Henry mummy wrap and the Henry finger sweep surgical techniques. J Sex Med 2009;6:619-22.

67. Sadeghi-Nejad H, Ilbeigi P, Wilson SK, Delk JR, Siegel A, Seftel AD, et al. Multi-institutional outcome study on the efficacy of closed-suction drainage of the scrotum in threepiece inflatable penile prosthesis surgery. Int J Impot Res 2005; 17:535-8.

68. LaPar DJ, Ailawadi G, Isbell JM, Crosby IK, Kern JA, Rich JB, et al. Mitral valve repair rates correlate with surgeon and institutional experience. J Thorac Cardiovasc Surg 2014;148:9951003; discussion 1003-4.

69. Sgroi MD, Darby GC, Kabutey NK, Barleben AR, Lane JS 3rd, Fujitani RM. Experience matters more than specialty for carotid stenting outcomes. J Vasc Surg 2015;61:933-8.

70. Cahill PJ, Pahys JM, Asghar J, Yaszay B, Marks MC, Bastrom $\mathrm{TP}$, et al. The effect of surgeon experience on outcomes of surgery for adolescent idiopathic scoliosis. J Bone Joint Surg Am 2014;96:1333-9.

71. Oberlin DT, Matulewicz RS, Bachrach L, Hofer MD, Brannigan RE, Flury SC. National practice patterns of treatment of erectile dysfunction with penile prosthesis implantation. J Urol 2015;193:2040-4.

72. McAbee KE, Pearlman AM, Terlecki RP. Infection following penile prosthesis placement at an academic training center remains low despite involvement of surgeons-in-training. Investig Clin Urol 2018;59:342-7.

73. Onyeji IC, Sui W, Pagano MJ, Weinberg AC, James MB, Theofanides MC, et al. Impact of surgeon case volume on reoperation rates after inflatable penile prosthesis surgery. J Urol 2017;197:223-9.

74. Caire AA, Boonjindasup A, Hellstrom WJ. Does a replacement or revision of an inflatable penile prosthesis lead to decreased patient satisfaction? Int J Impot Res 2011;23:39-42. 
75. Brant MD, Ludlow JK, Mulcahy JJ. The prosthesis salvage operation: immediate replacement of the infected penile prosthesis. J Urol 1996;155:155-7.

76. Mulcahy JJ. Long-term experience with salvage of infected penile implants. J Urol 2000;163:481-2.

77. Köhler TS, Modder JK, Dupree JM, Bush NC, McVary KT. Malleable implant substitution for the management of penile prosthesis pump erosion: a pilot study. J Sex Med 2009;6:1474-8.
78. Kaufman JM, Kaufman JL, Borges FD. Immediate salvage procedure for infected penile prosthesis. J Urol 1998;159:8168.

79. Knoll LD. Penile prosthetic infection: management by delayed and immediate salvage techniques. Urology 1998;52:287-90.

80. Habous M, Farag M, Williamson B, Laban O, Mahmoud S, Abdelwahab $\mathrm{O}$, et al. Conservative therapy is an effective option in patients with localized infection after penile implant surgery. J Sex Med 2016;13:972-6. 